Lucrările Seminarului Geografic Dimitrie Cantemir

Vol. 44, October 2017, pp. 85-100

http://dx.doi.org/10.15551/lsgdc.v44i0.06

\title{
Bioraffineries rurales : la question de l'ancrage territorial
}

Miravo Rakotovao ${ }^{1}$, Julie Gobert ${ }^{1}$, Sabrina Brullot ${ }^{1}$

${ }^{1}$ Université de Technologie de Troyes, France

To cite this article: Rakotovao, M., Gobert, J., Brullot, S. (2017). Bioraffineries rurales : la question de l'ancrage territorial. Lucrările Seminarului Geografic Dimitrie Cantemir, Vol. 44, pp. 85-100. DOI: $10.15551 /$ lsgdc.v44i0.06

To link to this article: http://dx.doi.org/10.15551/lsgdc.v44i0.06 


\title{
BIORAFFINERIES RURALES : LA QUESTION DE L'ANCRAGE TERRITORIAL
}

\author{
Miravo Rakotovao ${ }^{1}$, Julie Gobert ${ }^{2}$, Sabrina Brullot $^{3}$
}

\begin{abstract}
Résumé. Les bioraffineries sont des complexes industriels ayant pour vocation la transformation de la biomasse en une vaste gamme de produits bio-sourcés (alimentation humaine, alimentation animale, produits chimiques, matériaux) et d'énergie (carburants, chaleur et électricité) commercialisables (International Energy Agency, 2009). Ces installations semblent être en mesure d'apporter une contribution à la résolution des enjeux climatiques actuels, notamment la raréfaction des énergies fossiles ainsi que les émissions de gaz à effet de serre. Leur implantation en milieu rural nous amène cependant à nous interroger quant à leurs impacts économiques et sociaux sur le territoire de production. En effet, avant de produire les effets escomptés à l'échelle globale, il est une évidence que leurs retombées se font avant tout ressentir au niveau local. Dans cette perspective, l'objet de cet article consiste à appréhender les modalités de l'ancrage territorial de ce type de projet, et ce, dans le but de déduire les différents enjeux territoriaux qui en découlent. Pour éclairer le concept du bioraffinage, une description des bioraffineries est donnée au préalable, suivie d'une délimitation du territoire d'étude. Finalement, une revue de la littérature sur l'ancrage territorial en milieu rural a été effectuée dans le but d'inventorier les dynamiques de l'ancrage d'un projet agro-industriel de type bioraffinerie.
\end{abstract}

Mots-clés : bioraffineries rurales - territoire - ancrage territorial - impacts socioéconomiques - évaluation.

\section{Introduction}

Ces dernières décennies, la transition énergétique est devenue une préoccupation internationale. Face à des ressources fossiles qui s'épuisent, auxquelles se conjugue la lutte contre les émissions de gaz à effet de serre ; recourir à des énergies à la fois plus propres et disponibles dans le long terme devient capital. La biomasse semble répondre à ces critères. Non seulement constitue-t-elle une ressource renouvelable, mais ses origines sont aussi multiples. Si la transformation de la biomasse en énergie n'est pas chose nouvelle, sa valorisation de manière à en extraire plusieurs variétés de produits n'a été exercée que récemment. C'est l'objet même des bioraffineries. Par définition, la bioraffinerie est un complexe de transformation de la biomasse en un large spectre de produits et d'énergies à haute valeur ajoutée, de manière à valoriser toutes les composantes de la plante (Soetaert, 2009). Ainsi, une variété de technologies est mise en œuvre pour la production de

\footnotetext{
${ }^{1}$ Doctorante contractuelle chargée d'enseignement à l'Université de Technologie de Troyes

${ }^{2}$ Chercheure, Université de Technologie de Troyes ; chercheure associée au Lab'Urba et au LEESU

${ }^{3}$ Enseignante - chercheure à l'Université de Technologie de Troyes
} 
biocarburants, de produits chimiques, de produits d'alimentation humaine et animale, de biomatériaux, ainsi que de la chaleur et de l'électricité.

De par leur système d'approvisionnement, deux modèles de bioraffineries ont été distingués : les bioraffineries portuaires et les bioraffineries rurales (Jarry et Thomas, 2012 ; Ceapraz et al., 2016). Alors que les premières transforment de la biomasse principalement importée ; les deuxièmes utilisent essentiellement de la biomasse produite sur le territoire local. Comparées aux bioraffineries portuaires, les bioraffineries rurales témoignent d'un rapport plus fort au territoire local; en particulier, à travers l'échange de flux avec les agriculteurs locaux. Elles procurent de nouveaux débouchés aux exploitants agricoles et créent des liens entre l'industrie et l'agriculture (Papendiek et al., 2012). Par ailleurs, Lopilito et al. (2011) soutiennent qu'en plus de représenter une source de revenus pour les agriculteurs, ces bioraffineries créent de nouvelles opportunités pour les secteurs non agricoles. En d'autres termes, les bioraffineries des champs ${ }^{4}$ semblent contribuer au développement économique rural des territoires où elles sont implantées.

Cependant, les impacts socio-économiques de la bioraffinerie sur son territoire d'accueil ne peuvent être limités à ces seuls indices économiques. En effet, les enjeux territoriaux sont plus complexes et embrassent des domaines à la fois économiques, sociaux, institutionnels et culturels. Considéré comme un système complexe (Moine, 2006), le territoire englobe des réalités économiques et sociales mais aussi des formes idéologiques et politiques (Di Méo, 1991) qui lui sont propres. Granovetter (1985) soutient que dans le cadre de la société industrielle moderne, l'activité économique est ancrée dans des structures de relations sociales dans lesquels les acteurs ne se comportent pas (ou ne décident pas) en tant qu'atomes en dehors du contexte social. D'ailleurs, Sauvée et Viaggi (2016) affirment que le potentiel de développement des bioraffineries implantées en milieux ruraux est fortement lié à leur capacité à articuler l'aspect économique avec les dimensions sociales et environnementales de ceux-ci. Dans cette optique, en tant qu'installation agro-industrielle territorialisée, la bioraffinerie est mue par les dynamiques territoriales, et la prise en considération de celles-ci détermine le degré de son ancrage territorial. En effet, d'après Boons et Howard-Grenville (2009), l'ancrage d'une organisation fait référence à la contextualisation des activités économiques et organisationnelles au cœur d'arrangements et de processus d'ordre sociaux. L'enjeu est d'autant plus important que le développement économique de la bioraffinerie rurale est fortement conditionné par l'accessibilité et la disponibilité des ressources naturelles du territoire. Comme l'atteste Colletis (2010), « le développement d'une technologie dépend fondamentalement des facteurs économiques de contexte (disponibilité des ressources, prix relatifs, répartition des revenus, etc.) ». De même, Fourcade (2008) défend que «l'accès aux ressources agricoles impose souvent un ancrage territorial ». Frayssignes (2005) mentionne pour sa part, que l'ancrage territorial ne résulte pas uniquement d'un choix d'optimisation mais se réalise au gré des opportunités et des contraintes locales.

En outre, il est bien reconnu que l'échelle locale est fortement imbriquée dans le système global. En effet, « le territoire est emboîté dans un autre ensemble d'espaces qu'il influence et qui l'influencent réciproquement» (Leloup et al., 2005). Bon nombre de décisions, économiques ou politiques, prises au niveau macro (national, européen ou mondial) émanent de faits et observations à l'échelle micro. Force est de constater qu'avant

\footnotetext{
${ }^{4}$ Les bioraffineries rurales sont aussi appelées bioraffineries des champs.
} 
de produire les impacts environnementaux escomptés au niveau mondial, les unités de bioraffineries génèrent avant tout des impacts (positifs et négatifs) à l'échelle de la production, et plus largement, du territoire d'implantation. Si certains chercheurs y voient une opportunité de favoriser le développement économique rural, d'autres le perçoivent comme une menace à la viabilité même du secteur agricole. Selon Nieddu (1998), la régression de l'agriculture est due à l'industrialisation de l'alimentation, « faisant glisser la production de biens de consommation à la production de biens intermédiaires ». D'ailleurs, les controverses scientifiques autour de la question de la sécurité alimentaire et de la sécurité énergétique sont bien connues (Rosegrant, 2008; Mitchell, 2008 ; Zhang et al., 2010 ; Babcock, 2011 ; Ajanovic, 2011). Cependant, cette étude ne vise en rien à se positionner sur la question, mais plutôt à défendre la nécessité de cerner et contrôler les effets des différentes décisions politiques à différentes échelles, de manière à veiller à un développement durable effectif.

De ce fait, cet article se propose d'étudier les modalités probables de l'ancrage territorial d'une bioraffinerie rurale. Une description du territoire de la bioraffinerie sera entreprise. Par la suite, à travers une revue de la littérature, les dynamiques de l'ancrage territorial d'une activité économique en général, et d'un projet agro-industriel de type bioraffinerie en particulier seront étudiées ; et ce, afin de déduire les enjeux territoriaux qui en découlent.

\section{Bioraffineries rurales : de quoi parle-t-on ?}

\subsection{Le concept de bioraffinerie}

Alors que le bioraffinage est une technologie récente, qui se trouve encore entre la phase de lancement et de développement (Hellsmark, 2014 cité in Jacobson, 2015), différentes définitions du concept ont d'ores et déjà fait leur apparition. La plus connue d'entre elles a été donnée par l'IEA $^{5}$, qui décrit la bioraffinerie comme un complexe industriel portant sur la transformation de façon durable de la biomasse, en un large spectre de produits (alimentation humaine, alimentation animale, matériaux, produits chimiques) et d'énergie (carburants, chaleur, électricité) commercialisables. Une bioraffinerie peut utiliser toutes sortes de biomasse, qu'elles soient forestières, agricoles, aquacoles, de même que les déchets industriels et ménagers, les déchets organiques et la biomasse algale (de Jong et Jungmeier, 2015). Cependant, une distinction est de rigueur entre biomasse traditionnelle et biomasse moderne. Le terme «biomasse moderne» n'est autre que la biomasse traditionnelle utilisée grâce à des technologies de combustion efficaces et propres. Elle exclut les utilisations traditionnelles de la biomasse comme bois de chauffage (Demirbas, 2010).

Pour sa part, Cherubini (2010) définit la bioraffinerie comme une installation (ou un réseau d'installations) qui met en œuvre un ensemble de procédés pour convertir la biomasse en biocarburants, énergie et produits chimiques. Ce concept est analogue à la raffinerie de pétrole d'aujourd'hui, qui produit de multiples combustibles et produits du pétrole. D'après Kamm et Kamm (2004), la palette de produits d'une raffinerie végétale inclut non seulement

\footnotetext{
${ }^{5}$ Inernational Energy Agency
} 
les produits transformés dans une raffinerie pétrolière, mais aussi et surtout, les produits qui ne sont pas accessibles au sein de celle-ci.

\subsection{Classification des bioraffineries}

Les bioraffineries revêtent un caractère hétérogène. Il n'existe pas une seule et unique manière de les classifier du fait qu'elles utilisent une variété de matières premières, suivant des modèles d'approvisionnement différents; de même qu'une variété de technologies pour produire une variété de produits et d'énergies. Les principaux critères de classification rencontrés dans la littérature sont: (i) la nature des ressources végétales utilisées (Laurent et al., 2011 ; Naik et al., 2010 ; Cherubini et al., 2009) ; (ii) le type de produits transformés (Laurent et al., 2011 ; Cherubini et al., 2009) ; (iii) le type de procédé de conversion appliqué ( Cherubini, 2009). Le tableau ci-après présente de manière synthétique une caractérisation des bioraffineries suivant le type de ressource végétale utilisée, la catégorie de biomasse valorisée, ainsi que la nature des produits transformés (Tableau 1).

Tableau 1 : Typologie des bioraffineries

\begin{tabular}{|c|c|c|}
\hline Critère & Type & Description \\
\hline \multirow{4}{*}{$\begin{array}{l}\text { Ressources } \\
\text { végétales } \\
\text { utilisées }\end{array}$} & Bioraffineries vertes & $\begin{array}{l}\text { Biomasse humide : herbe, luzerne, trèfle, céréales } \\
\text { immatures ou ressources périssables (pommes de } \\
\text { terre, betteraves sucrières, etc.) }\end{array}$ \\
\hline & Bioraffineries céréalières & Céréales sèches (maïs, blé, riz) \\
\hline & $\begin{array}{c}\text { Bioraffineries } \\
\text { oléagineuses }\end{array}$ & $\begin{array}{l}\text { Graines de type oléagineuses (colza, tournesol, soja, } \\
\text { etc.) }\end{array}$ \\
\hline & $\begin{array}{c}\text { Bioraffineries } \\
\text { lignocellulosiques }\end{array}$ & $\begin{array}{l}\text { Ressources issues du bois et de toute biomasse } \\
\text { lignocellulosique (paille, bambou, miscanthus, etc.) }\end{array}$ \\
\hline \multirow{3}{*}{$\begin{array}{l}\text { Catégorie de } \\
\text { matières } \\
\text { premières }\end{array}$} & $\begin{array}{l}\text { Bioraffineries de } 1^{\text {ère }} \\
\text { génération }\end{array}$ & Plantes alimentaires (biomasse agricole) \\
\hline & $\begin{array}{l}\text { Bioraffineries de } 2^{\text {eme }} \\
\text { génération }\end{array}$ & $\begin{array}{l}\text { Matériaux lignocellulosiques (biomasse } \\
\text { lignocellulosique) }\end{array}$ \\
\hline & $\begin{array}{l}\text { Bioraffineries de } 3^{\text {ème }} \\
\text { génération }\end{array}$ & Algues (biomasse marine) \\
\hline \multirow{2}{*}{$\begin{array}{l}\text { Produits } \\
\text { transformés }\end{array}$} & $\begin{array}{l}\text { Bioraffineries centrées } \\
\text { sur les produits bio- } \\
\text { sourcés }\end{array}$ & $\begin{array}{l}\text { - Biomasse fractionnée en de multiples produits } \\
\text { bio-sourcés avec une valeur ajoutée maximale ; } \\
\text { - Résidus du procédé utilisés dans la production de } \\
\text { chaleur et d'électricité. }\end{array}$ \\
\hline & $\begin{array}{c}\text { Bioraffineries axées sur } \\
\text { l'énergie }\end{array}$ & $\begin{array}{l}\text { - Biomasse utilisée pour la production de } \\
\text { biocarburants, de chaleur et d'électricité ; } \\
\text { - Résidus du procédé vendus comme alimentation } \\
\text { animale ou valorisés comme produits à haute } \\
\text { valeur ajoutée. }\end{array}$ \\
\hline
\end{tabular}


En dehors de ces typologies classiques, la localisation géographique joue également un rôle prépondérant dans la distinction des bioraffineries, notamment, au regard de la logique économique véhiculée en leur sein. Si les bioraffineries situées dans les territoires portuaires se basent sur une logique d'agglomération de ressources et une économie d'échelle, les bioraffineries des champs, quant à elles, se fondent sur une logique de proximité de la demande et de synergie entre les acteurs (Cepreaz et al., 2016). Gobert (2015) expose que la bioraffinerie rurale se caractérise par l'importance de son processus d'intégration dans la mesure où elle est amenée à entretenir des liens plus ou moins étroits avec son territoire. Cette intégration s'étend à la région agricole d'approvisionnement et aux relations avec le monde agricole (Jarry et Thomas, 2012), mais pas seulement... En effet, les bioraffineries rurales peuvent s'appuyer sur des synergies avec divers acteurs publics-privés ainsi que des chercheurs et différentes communautés qui permettent la transformation et le développement d'un territoire (Cepreaz et al., 2016).

Par ailleurs, il sied de souligner, que récemment, de nouvelles formes de bioraffineries ont fait leur apparition ; ce sont les bioraffineries dites urbaines (Richel et al., 2016; Satchatippavarn et al., 2016). Ces bioraffineries se distinguent non seulement au regard de leur localisation géographique, mais aussi par rapport à la nature de la biomasse transformée en leur sein. Compte tenu des multiples flux de déchets urbains, elles visent à trouver la meilleure combinaison de processus pour récupérer ou convertir les molécules contenues dans chaque fraction de ces flux afin d'obtenir de l'énergie et des matériaux (Satchatippavarn et al., 2016).

Ces différentes typologies nous permettent de nous rendre compte du caractère hétéroclite du concept de bioraffinerie. En effet, leur rapport au territoire dépend d'un certain nombre de facteurs. Selon Gobert et Brullot (2012), « les bioraffineries s'inscrivent dans des territoires très divers et leur lien à leur environnement d'accueil dépend de l'aire d'approvisionnement de la ou des matières transformées, de la manière dont le projet de transformation de la biomasse a émergé et de la gouvernance du projet ».

\section{Ancrage territorial des bioraffineries rurales}

\subsection{Un territoire, mais lequel ?}

Il ne serait pas rationnel d'aborder la question de l'ancrage territorial sans passer par la description du territoire au sein duquel cet ancrage est amené à se produire. Le caractère plurivoque de la notion de territoire nécessite effectivement de délimiter de manière succincte la circonscription de l'étude. Comme l'affirme Bigo (2011), l'identité territoriale dépend de la clarté de son enveloppe frontalière. Cette section porte ainsi sur cette clarification du territoire d'étude. Sous l'angle de la géographie, le territoire a une localisation, une dimension, une forme, des caractéristiques physiques, des propriétés, des contraintes et des aptitudes (Elisslade, 2002). Pour décrire notre territoire, nous nous référerons dans un premier temps à sa dimension économique, puis à sa forme politicoadministrative. En effet, d'après Dumont (1999), un territoire s'aménage à travers les décisions des institutions publiques et celles des agents économiques. Bien que l'instance économique propre à toute formation sociale fournisse l'impulsion des fondations territoriales, elle requiert quasi simultanément, pour assurer son organisation et autoriser sa régulation, l'intervention d'un pouvoir politique (Di Méo, 1991). 
D’un point de vue purement économique, le territoire est défini comme un espace de ressources potentielles devant être révélées (Colletis, 2010); un point de vue que partage Frayssignes (2005) qui le décrit comme un support, une ressource dont l'appropriation s'effectue à travers les activités économiques, c'est-à-dire les activités humaines qui utilisent ses potentialités. De même, Leloup et al. (2005) perçoivent le territoire comme « une entité active qui puise son potentiel de développement dans ses ressources locales, entendues au sens large, c'est-à-dire avec ses activités, ses acteurs, ses réseaux ». Courlet et al. (1993), pour leur part, identifient de façon plus claire la nature de ces ressources : le territoire est pour eux, un stock de ressources matérielles et immatérielles détenues et gérées par différents acteurs (entreprises, institutions de recherche et de formation, pouvoirs publics locaux, etc.). Au regard de la littérature, les acteurs économiques décident donc de s'implanter au sein de ces territoires principalement en raison de la disponibilité et de l'accessibilité des ressources. Aussi, cette logique d'implantation conditionne-t-elle leur compétitivité économique. D’après Sergent et Cazals (2015), les critères qui déterminent la compétitivité d'une implantation locale par rapport à une autre sont liés au coût de la main d'œuvre, au coût de l'énergie, aux contraintes environnementales, aux coûts du transport mais aussi et surtout à la qualité et au coût de l'approvisionnement. Dans cette perspective, le territoire de production constitue l'angle pertinent pour apprécier les impacts socioéconomiques de la bioraffinerie à l'échelle microéconomique. Or, la mise en œuvre de la production implique à la fois une combinaison de transformations physiques et la participation de plusieurs acteurs tout au long de la chaîne (UNIDO $\left.{ }^{6}, 2009\right)$. Les principaux acteurs qui interviennent dans la chaîne de valeur ${ }^{7}$ de la bioraffinerie sont alors les producteurs de biomasse, les fournisseurs industriels, les transporteurs, les employés, les clients industriels ainsi que les utilisateurs finaux.

Politiquement parlant, le territoire est aussi défini comme un espace circonscrit par des frontières constituant la limite de l'exercice du pouvoir d'une entité souveraine sur la population (Bigo, 2011). Si cette souveraineté revient a priori à la nation, en Europe, et en France en particulier, elle a depuis été découpée en plusieurs couches administratives. Au niveau du territoire français, il y a souvent un minimum de six échelons administratifs à considérer: la commune, l'intercommunalité, le département, la région, l'État et l'Union européenne, chacune ayant ses responsabilités propres définies par des textes (Dumont, 1999). Dans notre approche, nous estimons que la clé de lecture adéquate pour étudier les impacts économiques et sociaux des bioraffineries est le territoire régional. Premièrement, les régions doivent être en capacité d'agir en tant que modérateurs des intérêts locaux, en formant des liens entre différents partenaires dans une sphère commune d'action régionale, et en négociant leurs intérêts avec le centre (Bullmann, 1997, cité in Perraud, 2016). En

\footnotetext{
${ }^{6}$ United Nations Industrial Development Organization

${ }^{7}$ La chaîne de valeur est un concept pouvant être simplement décrit comme l'ensemble de la gamme d'activités requises pour amener un produit de l'étape initiale d'approvisionnement en intrants, passant par les différentes phases de la production, jusqu'à sa destination finale sur le marché. Sa définition d'origine a été fournie par Kaplinsky et Morris (2000): "The value chain describes the full range of activities which are required to bring a product or service from conception, through the different phases of production (involving a combination of physical transformation and the input of various producer services), delivery to final consumers, and final disposal after use."
} 
outre, les lois $\mathrm{NOTRE}^{8}$ et $\mathrm{MAPTAM}^{9}$ portant sur la réforme territoriale, ont concentré la compétence économique aux régions, bien que l'échelle départementale reste intéressante sur les sujets agricoles et industriels (les départements pouvant, dans le cadre d'une convention avec la région, abonder les aides aux secteurs agricole, sylvicole et piscicole). Par conséquent, les données économiques (création d'emplois, revenus agricoles, développement économique rural, etc.) sont agrégées au niveau de la région. L'échelle régionale comme grille de lecture des impacts de la bioraffinerie rejoint également les objectifs de la Loi de Transition Energétique pour la Croissance Verte (LTECV) adoptée en août 2015, qui vise à rapprocher les objectifs nationaux des acteurs locaux (OIE, 2016) ${ }^{10}$. L'article 188 stipule ainsi que « la région constitue l'échelon pertinent pour coordonner les études, diffuser l'information et promouvoir les actions en matière d'efficacité énergétique $»^{11}$.

Cependant, en tant qu'acteur économique, la bioraffinerie est amenée à nouer des interactions avec divers acteurs publics-privés aussi bien à l'intérieur du territoire régional, qu'à l'extérieur. En effet, si la région agit sur une échelle administrative prédéfinie, les agriculteurs, les fournisseurs ou les clients industriels peuvent appartenir à d'autres régions administratives, voire, d'autres pays. Il est alors de rigueur de préciser que l'évaluation de l'ancrage dans la présente étude se cantonne à la région administrative au sein de laquelle la bioraffinerie est implantée. En d'autres termes, même si la chaîne de valeur de la bioraffinerie est étendue à d'autres échelles géographiques et/ou administratives, seuls les acteurs appartenant au territoire régional seront pris en considération, comme il est décrit dans la figure ci-après (Fig.1).

${ }^{8}$ LOI ${ }^{\circ}$ 2015-991 du 7 août 2015 portant nouvelle organisation territoriale de la République disponible en ligne sur https://www.legifrance.gouv.fr/affichTexte.do?cidTexte=JORFTEXT000030985460\&categorieLien=i d, consulté le 21 février 2017.

${ }^{9}$ LOI n ${ }^{\circ} 2014-58$ du 27 janvier 2014 de modernisation de l'action publique territoriale et d'affirmation des métropoles disponible en ligne sur https://www.legifrance.gouv.fr/affichTexte.do?cidTexte=JORFTEXT000028526298, consulté le 21 février 2017.

${ }^{10}$ Observatoire de l'Industrie Electrique

${ }^{11}$ LOI n ${ }^{\circ}$ 2015-992 du 17 août 2015 relative à la transition énergétique pour la croissance verte Article 188, disponible en ligne sur https://www.legifrance.gouv.fr/affichTexteArticle.do;jsessionid=6EFCB3B897B1D0286D87AD9B3 BD06A83.tpdila09v_2?idArticle=JORFARTI000031045729\&cidTexte=JORFTEXT000031044385\& dateTexte=29990101\&categorieLien=id, consulté le 07 février 2017. 


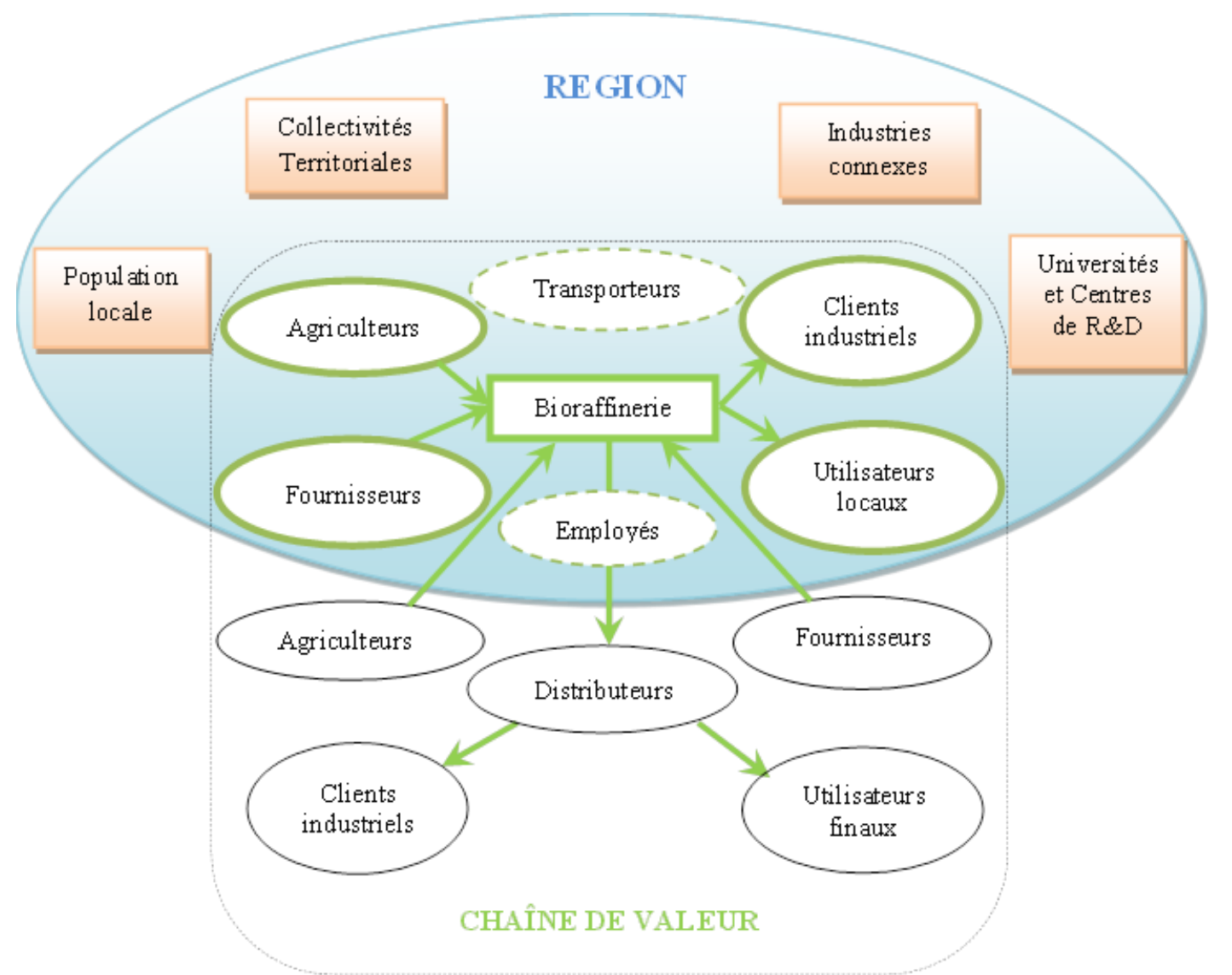

Figure 1 : Description du périmètre d'étude

\subsection{Les bioraffineries rurales dans le contexte de l'ancrage territorial}

Stricto sensu, l'ancrage renvoie à l'action d'immobiliser ou de stabiliser un bateau, de le fixer au moyen de son ancre. Pris au sens large, l'ancrage se réfère à l'action d' "établir solidement une institution quelque part ${ }^{12}$. En d'autres termes, il renvoie à l'implantation, l'enracinement ; s'ancrer, c'est « s'installer, s'enraciner dans un lieu, dans un contexte $»^{13}$. L'ancrage territorial d'une bioraffinerie se réfère alors à son enracinement dans le territoire. Toutefois, cet enracinement n'est pas que le résultat d'un processus physique car «ancrer», c'est aussi «fixer ou inculquer profondément un sentiment ${ }^{14}$. Ce qui nous

\footnotetext{
${ }^{12}$ Dictionnaire LAROUSSE disponible en ligne sur http://www.larousse.fr/dictionnaires/francais/ancrage/3341?q=ancrage\#3338, consulté le 08 février 2017.

${ }^{13}$ Le Dictionnaire disponible en ligne sur http://www.le-dictionnaire.com/definition.php?mot=ancrage, consulté le 08 février 2017.

${ }^{14}$ Dictionnaire LAROUSSE disponible en ligne sur http://www.larousse.fr/dictionnaires/francais/ancrer/3343?q=ancrer\#3340, consulté le 08 février 2017. 
renvoie à l'aspect intangible et immatériel de l'ancrage. En s'ancrant sur le territoire, la bioraffinerie est amenée à épouser les valeurs de celui-ci. Dans son approche, Frayssignes (2005) désigne l'ancrage territorial comme «l'ensemble des liens réciproques qui unissent une activité économique (acteur, entreprise, filière, ...) avec un territoire ». Boons et Howard-Grenville (2009), quant à eux, décrivent ce phénomène comme la contextualisation des activités économiques et organisationnelles au cœur d'arrangements et de processus d'ordre sociaux. En somme, l'ancrage territorial se fonde sur la coordination avec les acteurs du territoire. Par ailleurs, l'ancrage se caractérise entre autres, par le fait qu'il n'est pas ponctuel. Il ne s'opère pas qu'au moment de l'installation sur le territoire; au contraire, il s'inscrit dans le temps. Colletis et al. (1999) voient ce temps comme celui de la construction par l'acteur économique de son environnement territorial. Se pose alors la question suivante : De quoi l'environnement territorial de la bioraffinerie est-il constitué?

D'après Bertrand (1996), le phénomène d'ancrage repose sur des relations sociales et des coopérations entre acteurs locaux. De ce fait, l'environnement territorial de la bioraffinerie, tel que nous la décrivons, est constitué par l'environnement interne d'une part et l'environnement externe, d'autre part. Nous entendons par environnement interne, les acteurs qui sont directement rattachés à la chaîne de valeur. Ce sont les agriculteurs locaux ou producteurs de biomasse, les transporteurs, les employés de l'unité de bioraffinerie, les clients industriels et les utilisateurs finaux. Par ailleurs, l'environnement externe correspond aux parties prenantes du territoire avec lesquelles la bioraffinerie est amenée à interagir, notamment à travers des partenariats public-privés et des synergies. On distingue de ce fait, les collectivités territoriales, les industries connexes, les universités et les centres de recherche et développement, et finalement, la population locale.

\subsection{Quelles clés de lecture pour mesurer l'ancrage territorial des bioraffineries rurales?}

Différentes approches sont évoquées dans la littérature en matière de déterminants de l'ancrage territorial. Certaines études (Frayssignes, 2005 ; Fourcade, 2008) abordent la question de l'ancrage sous l'angle des dynamiques de proximité (Rallet et Torre, 2004 ; Boshma, 2004). Ce concept a été développé par l'école des proximités en 1993 (Torre, 2009). Il met en exergue le caractère à la fois temporel et spatial de l'ancrage en se déclinant sous forme de triptyque: (i) la proximité géographique qui fait référence à la proximité spatiale des emplacements physiques des organisations; (ii) la proximité organisationnelle décrivant les interactions entre acteurs et groupes d'acteurs au sein d'organisations formelles et informelles; et (iii) la proximité institutionnelle faisant référence à l'adhésion des parties prenantes à un ensemble de règles, de représentations et de valeurs qui guideront leurs stratégies et les conduiront à une coordination régulière du comportement (Balland et al., 2015, Frayssignes, 2005). Dans le cadre particulier de l'innovation, Boshma (2004) a étendu les composantes de la proximité en y greffant la proximité cognitive, se rapportant au partage de la même base de connaissance et de compétences entre les individus ; et la proximité sociale, traduisant l'impact des relations sociales sur les résultats économiques, notamment sur l'apprentissage collectif et l'innovation. Ainsi, les notions de proximités jouent un rôle de miroir à l'ancrage dans la mesure où elles représentent un potentiel que des individus ou des groupes peuvent mobiliser ou activer (Torre, 2010). En d'autres termes, le degré d'ancrage territorial de la bioraffinerie rurale dépend de la capacité de ceux qui la 
gèrent à mobiliser les différentes formes de proximité sus-citées. Toutefois, Boshma (2004) soutient qu'un excès ou une insuffisance de proximité portent préjudice à l'innovation ; « la proximité demande une certaine distance, mais qui ne soit pas trop grande, entre acteurs et organisations ».

Dans leur démarche, Boons et Howard-Grenville (2009) identifient six mécanismes de l'ancrage en écologie industrielle ${ }^{15}$, qu'ils nomment «mechanisms of embeddedness » : (i) «cognitive embeddedness » ou encastrement cognitif, qui renvoie à l'utilisation d'un certain nombre d'heuristiques pour prendre des décisions au jour le jour, tout en s'appuyant sur d'autres raccourcis lorsque les besoins en information ou la capacité cognitive à prendre des décisions «rationnelles» seraient accablantes; (ii) «cultural embeddedness» ou encastrement culturel, qui fait référence à l'influence des représentations collectives et des normes communes sur le comportement économique et organisationnel. Il diffère de l'encastrement cognitif du fait qu'il agit sur le collectif plutôt que l'individuel; (iii) «structural embeddedness» ou encastrement structurel, qui se rapporte à la contextualisation de l'échange économique dans des modèles relationnels continus (c'est-àdire que la structure des interactions sociales entre les acteurs façonnent les flux d'information et d'influence et forment les échanges économiques et autres formes d'échanges) ; (iv) «political embeddedness » ou encastrement politique, qui renvoie à la manière dont les institutions économiques et leurs décisions sont façonnées par une lutte pour le pouvoir, impliquant les acteurs économiques, les institutions non marchandes, en particulier l'Etat (collectivités territoriales), et les classes sociales; et finalement, (v-vi) l'encastrement spatial et temporel dit « spatial and temporal embeddedness », qui prend en compte la manière dont les interactions sont influencées par la proximité physique et le temps (la première est considérée comme un facteur catalyseur dans de nombreux domaines, notamment l'apprentissage, la construction de la confiance, etc. ; le temps est tout aussi important considérant que l'évolution des systèmes industriels implique généralement de longues périodes). En résumé, l'enracinement social témoigne de la nécessité d'une appropriation à la fois identitaire et institutionnelle du territoire (Frayssignes, 2005), et cette appropriation s'amplifie dans le temps et dans l'espace.

Pour sa part, Bertrand (1996) a identifié deux principaux facteurs de l'ancrage territorial, qui pour l'essentiel, constituent des facteurs intangibles ou immatériels. Le premier concerne les relations personnelles que l'acteur économique tisse et développe avec d'autres acteurs locaux, sachant que certaines d'entre elles constituent des coopérations créatrices de ressources. Le second facteur est celui de la création de ressources humaines. L'auteur qualifie ces ressources comme étant spécifiques par le fait qu'elles résultent de l'articulation de différentes compétences, s'appuyant sur des rapports de confiance et sur la réputation de chacun des protagonistes ; et par conséquent, ne peuvent être reproductibles sur d'autres espaces.

Dans le contexte précis des bioraffineries, une étude se rapportant à leur ancrage territorial a été initiée par Gobert et Brullot (2015). Les auteurs se sont ainsi interrogés quant à la capacité des bioraffineries à mobiliser les capitaux du territoire. Par définition, Les

${ }^{15}$ L'écologie industrielle est l'étude des flux de matières et d'énergie dans les activités industrielles et de consommation, l'effet de ces flux sur l'environnement ainsi que l'influence des facteurs économiques, politiques et sociaux sur l'utilisation, la transformation et l'élimination des ressources (White, 1994 cité dans Ayres, 2002). 
capitaux territoriaux représentent les actifs et les potentiels que l'on est en mesure d'exploiter à l'échelle du territoire (Camagni, 2008). Ils font référence au stock d'actifs mobilisables au sein d'une région ou d'une ville, constituant la base de leur développement endogène (OECD, 2001). Sept principaux capitaux ont alors été inventoriés et considérés mobilisables par un projet de bioraffinerie : le capital organisationnel, le capital culturel, le capital cognitif, le capital technique, le capital naturel, le capital institutionnel et la capital structurel. La figure ci-dessous (Fig.2) présente la description relative à chacun de ces capitaux.

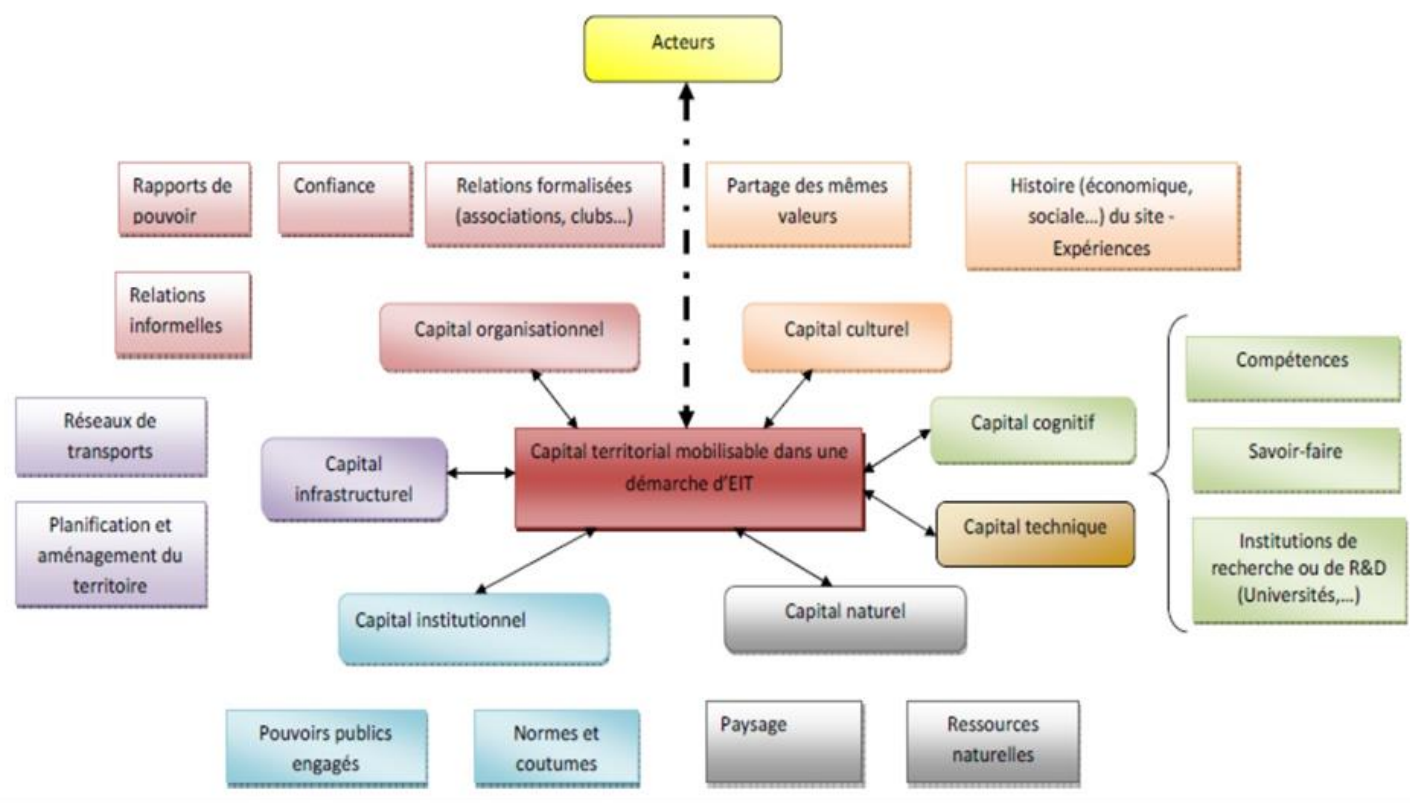

Figure 2 : Les composantes du capital territorial Source : Gobert et Brullot, 2015

Comparée aux précédentes, cette vision a la particularité de comptabiliser la mobilisation des flux matériels (ressources naturelles) comme étant l'un des déterminants du processus d'ancrage. Une réflexion que rejoignent Cerceau et al. (2013) en assimilant l'ancrage territorial des activités économiques à une volonté de s'engager davantage dans la meilleure prise en compte de l'environnement naturel en termes de gestion de ressources, mais aussi de l'environnement humain, à savoir les compétences locales. En effet, il sied de rappeler, d'une part, qu'en dépit du caractère économique de l'activité de la bioraffinerie, celle-ci participe peu ou prou, à travers la valorisation de la biomasse locale, à la construction du territoire. Or, dans une économie de création de ressources, l'objet ne consiste plus à se procurer ces ressources, mais également à en faire un meilleur usage (Bertrand, 1996). Aussi, l'utilisation de façon rationnelle des ressources matérielles du territoire constitue-t-elle un déterminant de l'ancrage territorial d'une activité économique. D'autre part, à côté des ressources matérielles, le territoire produit et mobilise des 
compétences, considérées, elles aussi, comme des ressources (Pecqueur et Ternaux, $2006^{16}$ ). Cerner l'ancrage territorial des bioraffineries rurales revient donc parallèlement à s'interroger sur la manière dont elles mobilisent, s'il y a lieu, les compétences locales.

Dans une étude se rapportant au développement des bioraffineries lignocellulosiques, Sergent et Cazals (2015) considèrent l'ancrage comme lié à l'organisation des chaînes de valeur sur un ou plusieurs territoires. De manière générale, le bioraffinage est un concept d'innovation qui vient se greffer à une industrie pré-existante, appartenant souvent à des multinationales (on peut citer l'implémentation d'une bioraffinerie lignocellulosique dans une industrie papetière, ou bien l'insertion d'une bioraffinerie agricole dans une industrie amidonnière). Dans ce cas, «l'ancrage territorial de la bioraffinerie prend un caractère multi-scalaire où les grandes régions du monde, les pays comme les localités, constituent des espaces d'affrontement des intérêts et de mise en concurrence ou au contraire de collaboration et d'échange ». D'après Gereffi et al. (2005), les acteurs du réseau, peuvent bénéficier des effets de transactions répétées, de la réputation et des normes sociales qui sont incorporées dans ces emplacements géographiques ou ces groupes sociaux particuliers. De même, Humphrey et Schmitz (2001) soutiennent que cette mutation industrielle peut actionner une remise à niveau aussi bien technique (produits et procédés) que fonctionnelle de la production. Toutefois, les bioraffineries s'implantent sur des territoires dont la nature des ressources et les conditions d'accès à celles-ci sont gouvernées par des dynamiques locales particulières. L'enjeu, pour les exploitants de la bioraffinerie, revient alors à «prendre en compte les différentes formes d'articulation entre le local et le global en s'appuyant sur les pratiques des acteurs à partir d'une analyse de la diversité de leurs logiques, qui peuvent s'opposer mais qui sont toutefois déterminantes de la diversité des formes d'ancrage territorial d'une industrie » (Sergent et Cazals 2015).

A l'issue de ces différentes approches qui, soulignons-le, ne constituent que des exemples parmi tant d'autres, nous pouvons déduire que le phénomène d'ancrage territorial requiert la considération d'un certain nombre de dimensions qui ne peuvent être réduites aux seules dimensions économiques. L'ancrage est à la fois spatial, dans la mesure où il prend en considération différents acteurs public-privés au sein de l'espace qu'est le territoire ; mais aussi temporel, par le fait qu'il met un certain temps à s'instaurer. L'ancrage revêt simultanément un caractère spécifique qui justifie sa non-reproductibilité au sein d'autres territoires. L'influence de l'ancrage d'organisations particulières dans des contextes particuliers doit être explorée avec le plus grand soin, au regard des objectifs visés (Boons et Howard-Grenville, 2009). L'ancrage territorial implique de ce fait une bonne connaissance du territoire d'implantation tout en gardant à l'esprit la spécificité de chaque territoire.

\section{Conclusion}

Après avoir globalement expliqué ce que sont que les bioraffineries, nous avons donné les différentes typologies usuellement rencontrées dans la littérature en la matière. En outre, nous avons présenté le contexte du territoire et de l'ancrage des bioraffineries rurales, notamment à travers la description de leur environnement territorial. Finalement, nous avons présenté différentes clés de lecture permettant de mesurer l'ancrage territorial d'un projet

\footnotetext{
${ }^{16}$ Cité in Bel (2009)
} 
agro-industriel territorialisé tel que la bioraffinerie rurale, et ce, dans le but d'appréhender les enjeux territoriaux qui émanent de ce processus.

Cet article permet donc de mettre en lumière les déterminants de l'ancrage territorial des bioraffineries rurales. Constituant des infrastructures fixées au sol, elles dépendent principalement des ressources naturelles du territoire. Toutefois, au-delà de l'utilisation de la biomasse locale pour alimenter leurs usines de production, ces installations se développement sur un territoire pourvu d'identité à la fois économique, politique, mais aussi sociale. Leur ancrage territorial se voit conditionné par leur capacité à prendre en considération ces différentes dimensions. Par conséquent, les exploitants des bioraffineries sont amenés à tisser des relations de coordination avec diverses parties prenantes du territoire (agriculteurs locaux et coopératives agricoles, collectivités locales, industries connexes, universités et centres de recherche et développement, etc.). Dans cette étude, qui fait l'objet d'une thèse en cours, l'échelle d'évaluation de l'ancrage constitue la région de production. L'objectif est de construire un modèle d'évaluation socio-économique de tels projets, qui intègre davantage les dynamiques du territoire au travers de l'analyse de cas d'étude français.

\section{Références bibliographiques}

1. Ajanovic A., 2011. Biofuels versus food production: Does biofuels production increase food prices? Energy, 36, 4, 2070-2076, Elevier.

2. Ayres R. Ayres L.W., 2002. A Handbook of Industrial Ecology, Cheltenham. UK \& Northampton, Massachusetts, Edward Elgar Publishing, 680p.

3. Babcock B.A., 2011. The impact of US Biofuel policies on agricultural price levels and volatility, Programme on Agricultural Trade and Sustainable Development, 35, Center of Agricultural and rural development, Iowa State University, 26p.

4. Balland P.A., Boschma R., Frenken K., 2015. Proximity and Innovation: From Statics to Dynamics. Regional Studies, 49, 6, 907-920.

5. Bel M., 2009. Compétences et dynamiques territoriales : quelles interactions ? Géographie, économie, société 2009/3, 11, 213-232, Lavoisier.

6. Bertrand N., 1996. L'ancrage spatial des entreprises en milieu rural: de l'espace fonctionnel à l'espace territoire. Thèse de doctorat ès économie appliquée. Université Pierre Mendès-France, Grenoble.

7. Bigo D., 2011. Frontières, territoire, sécurité, souveraineté. CERISCOPE Frontières, 1-13, URL : http://ceriscope.sciences-po.fr/content/part1/frontières-territoire-sécurité-souveraineté.

8. Boons F., Howard-Grenville J., 2009. The social embeddedness of Industrial Ecology, 281p. Edward Elgar Publishing Limited. British Library. United Kingdom.

9. Boschma R., 2004. Proximité et innovation, Économie rurale, 280, Proximité et territoires, 824, Creative commons.

10. Camagni R., 2008. Regional Competitiveness: Towards a Concept of Territorial Capital. Modeling Regional Scenarios for the Enlarged Europe. Springer Berlin Heidelberg. Part of the series Advances in Spatial Science, 33-47.

11. Ceapraz I.L., Kotbi G., Sauvée L., 2016. The territorial biorefinery as a new business model, Bio-based and applied economics 5, 1, 47-62, Firenze University Press.

12. Cerceau J., Donsimoni M., Labaronne D., Mat N., 2013. Ecologie industrielle dans les territoires portuaires du Maghreb, Presses des Mines, 213-236, HAL.

13. Cherubini F., 2010. The biorefinery concept: Using biomass instead of oil for producing energy and chemicals, Energy Conversion and Management, 51, 1412-1421, Elsevier. 
14. Cherubini F., Jungmeier G., Wellisch M., Willke T., Skiadas I., Van Ree R., de Jong E., 2009. Toward a common classification approach for biorefinery systems, Biofuels, bioproducts and biorefining, 3, 534-546, Society of Chemical Industry and John Wiley \& Sons, Ltd.

15. Colletis G., 2010. Co-évolution des territoires et de la technologie: une perspective institutionnaliste, Revue d'Economie Régionale et Urbaine, 2010/2, 235-249, Armand Colin.

16. Colletis G., Gilly J.P., Leroux I., Pecqueur B., Perrat J., Rychen F., Zimmermann J.B., 1999. Construction territoriale et dynamiques productives, Document de travail.

17. Courlet C., Pecqueur B., Soulage B., 1993. Industrie et dynamiques de territoire, Revue d'économie industrielle, 64, 2, 7-21, Creative commons.

18. De Jong E., Jungmeier G., 2015. Biorefinery Concepts in Comparison to Petrochemical Refineries. Industrial Biorefineries and White Biotechnology, 3-33, Elsevier.

19. Demirbas A., 2010. Biorefineries, For Biomass Upgrading Facilities, Green Energy and Technology. Springer. 240p.

20. Di Méo G., 1991. La genèse du territoire local : complexité dialectique et espace-temps, Annales de Géographie 100, 559, 273-294. Persée.

21. Dumont G.F., 1999. L'organisation administrative de l'espace et l'aménagement du territoire: Pour une nouvelle méthode, Conférence universitaire de démographie et d'étude des populations, 17-24, HAL.

22. Elissalde B., 2002. Une géographie des territoires, L'information géographique, 66, 193-205, Persée.

23. Fourcade C., 2008. Des dynamiques de proximité innovantes: le cas des systèmes agroalimentaires localisés en France, Cahiers Agricultures, 17, 6, 520-525.

24. Frayssignes J., 2005. Les AOC dans le développement territorial Une analyse en termes d'ancrage appliquée aux cas français des filières fromagères, Thèse, Mention Géographie, Institut National Polytechnique de Toulouse, Université de Toulouse, France, 469p.

25. Gereffi G., Humphrey J., Sturgeon T., 2005. The governance of global value chains, Review of International Political Economy 12, 1, 78-104.

26. Gobert J., 2015. The territorial biorefinery: An embedded socio-technical system of innovation, ERSA Conference. Lisbon. August, 27-29.

27. Gobert J., Brullot S., 2012. Bioraffineries: une logique éco-industrielle et territoriale? Ecologie industrielle et territoriale, Colloque Interdisciplinaire sur l'Ecologie Industrielle et Territoriale, 253-268, Presses des Mines.

28. Gobert J., Brullot S., 2017. La mobilisation du capital territorial pour le développement d'une logique d'EIT, Revue d'Economie Régionale et Urbaine (Accepté, à paraître).

29. Granovetter M., 1985. Economic action and social structure: The problem of embeddedness, American Journal of Sociology, 91, 3, 481-510, University of Chicago Press, 2002.

30. Humphrey J., Schmitz H., 2002. How does insertion in global value chains affect upgrading in industrial clusters? Regional Studies, 36, 9, 1017-1027, Institute of Development Studies, University of Sussex, Brighton.

31. International Energy Agency, 2009. Biorefineries: adding value to sustainable utilization of biomass. Task 42 Biorefinery.

32. Jacobson A., 2015. Socio-economic assessment of implementing mobile biorefineries, a prestudy with a focus on the European Union, Master's thesis, Department of Energy and Environment, Chalmers University of Technology, Sweden, 61p.

33. Jarry B., Thomas D., 2012. Les alternatives végétales aux ressources fossiles : concept et enjeu territorial, Les entretiens IAR, Bioraffinerie internationale, Première édition.

34. Kamm B., Kamm M., 2004. Principles of biorefineries, Epub, Appl Microbiol Biotechnol. 64, 2, 137-45. Springer-Verlag.

35. Kaplinsky R., Morris M., 2000. A handbook for value chain research, 109p. 
36. Laurent P., Roiz J, Wertz J.L., Richel A., Paquot M., 2011.Le bioraffinage, une alternative prometteuse à la pétrochimie, Biotechnol. Agron. Soc. Environ., 15, 4, 597-610, Wallonie.

37. Leloup F., Moyart L., Pecqueur B., 2005. La gouvernance territoriale comme nouveau mode de coordination territoriale? Géographie, économie, société, 2005/4, 7, 321-332, Lavoisier.

38. Lopolito A., Morone P., Sisto R., 2011. Innovation niches and socio-technical transition: A case study of bio-refinery production, Futures, 43, 27-38, Elsevier.

39. Mitchell D., 2008. A note on rising food price, Policy Research Working Paper, 4682, 20p., World Bank Development Prospects Group.

40. Moine A., 2006. Le territoire comme un système complexe : un concept opératoire pour l'aménagement et la géographie, L'Espace géographique, 2, 35, 115-132. Belin.

41. Naik S.N., Goud V., Rout P., Dalai A., 2010. Production of first and second generation biofuels: a comprehensive review, Renewable and Sustainable Energy Reviews 14, 578-597, Elsevier.

42. Nieddu M., 1998. Dynamique de longue période dans l'agriculture productive et mutations du système agro-industriel français contemporain, Thèse es sciences économique, Faculté de Sciences Economiques et de Gestion, Université de Reims, France, 504p.

43. Observatoire de l'Industrie Electrique, 2015. LOI $n^{\circ}$ 2015-992 du 17 août 2015 relative à la transition énergétique pour la croissance verte, Article 188, disponible en ligne sur https://www.legifrance.gouv.fr/affichTexteArticle.do;jsessionid=6EFCB3B897B1D0286D87AD9B3 BD06A83.tpdila09v_2?idArticle=JORFARTI000031045729\&cidTexte=JORFTEXT000031044385\& dateTexte $=29990101 \&$ categorieLien=id , consulté le 07 février 2017.

44. Organization for Economic Co-operation and Development, 2001. OECD Territorial Outlook. Territorial Economy. OECD Publications Service.

45. Papendiek F., Ende H.-P., Steinhardt U., Wiggering H., 2012. Biorefineries: Relocating Biomass Refineries to the Rural Area. IALE D. Landscape Online 27, 1, 1-9, Iale, Deutschland.

46. Perraud D., 2016. Les politiques agricoles et rurales dans les régions: une nouvelle organisation des pouvoirs publics en Europe ? Economie rurale, 261, 7-22, Creative commons.

47. Rallet A., Torre A., 2004. Proximité et localisation, Economie rurale, 280, Proximité et territoires, 25-41, Creative commons.

48. Richel A., Haubruge E., Jacquet N., 2016. Production of biofuels and biobased compounds in urban biorefineries: a strategy to manage waste in Wallonia? $6^{\text {th }}$ International Conference on Engineering for Waste and Biomass Valorization, May 23-26, Albi, France.

49. Rosegrant M.W., 2008. Biofuels and Grain Prices: Impacts and Policy Responses. International Food Policy Research Institute, 1-4, Washington, DC.

50. Satchattipavarn S., Matrinez-Henandez E., Hang M., Leach M., Yang A., 2016. Urban biorefineries for waste processing, Chemical Engineering Research and Design, 107, 81-90, Elsevier.

51. Sauvée L., Viaggi D., 2016. Biorefineries in the bio-based economy: opportunities and challenges for economic research, Bio-based and applied economics, 5, 1, 1-4, Firenze University Press.

52. Sergent A., Cazals C., 2015. L'industrie papetière face au développement de la bioraffinerie lignocellulosique. Dynamiques institutionnelles et perspectives territoriales, Economie rurale, 349350, 13-30, Société Française d'Économie rurale.

53. Soetaert W., 2009. Defining biorefineries and different concepts. Biorefinery Euroview. Biopol.http://www.biorefinery.nl/fileadmin/biopol/user/documents/ws2009/04_Wim_Soetaert_presen tation_BioreFuture_2009.pdf. Accessed 3 November 2016.

54. Torre A., 2009. Retour sur la notion de Proximité Géographique, Géographie, économie, société 2009/1, 11, 63-75, Lavoisier.

55. Torre A., 2010. Jalons pour une analyse dynamique des Proximités, Revue d'Économie Régionale \& Urbaine, 2010/3, 409-437, Armand Colin.

56. United Nations Industrial Development Organization, 2009. Agro-value chains analysis and development: The UNIDO approach, A staff working paper, Vienne, Autriche, 74p. 
57. Zhang Z., Lohr L., Escalante C., Wetzstein M., 2010. Food versus fuel: what do prices tell us? Energy Policy, 38, 445-451, Elsevier. 